\title{
Adiponectin and markers of metabolic syndrome in obese children and adolescents: impact of 8-mo regular physical exercise program
}

\author{
Henrique Nascimento ${ }^{1,2}$, Elísio Costa ${ }^{1,2}$, Susana Rocha ${ }^{1,2}$, Clarice Lucena ${ }^{3,4}$, Petronila Rocha-Pereira ${ }^{5}$, Carla Rêgo ${ }^{6}$, \\ Helena Ferreira Mansilha ${ }^{7}$, Alexandre Quintanilha ${ }^{2,8}$, Luísa Aires ${ }^{3,4}$, Jorge Mota ${ }^{3}$, Alice Santos-Silva ${ }^{1,2}$ and Luís Belo ${ }^{1,2}$
}

BACKGROUND: Adiponectin circulates as low-, medium-, and high-molecular-weight multimers (LMW, MMW, and HMW) and influences lipid profile and insulin resistance (IR), HMW being considered as the most biologically active form. We aimed to study the relation between adiponectin and markers of metabolic syndrome (MS) in pediatric obesity, and the impact of physical exercise.

METHODS: The study consisted of a cross-sectional part and an 8-mo physical exercise program. Lipid profile, insulin, glucose, C-reactive protein (CRP), total adiponectin (TA), and homeostasis model assessment IR (HOMA-IR) were measured. Adiponectin multimers were studied in a prepubertal group.

RESULTS: Obesity is associated with increased dyslipidemia, $I R$, and inflammation. TA is correlated inversely with adiposity, triglycerides, HOMA-IR, and CRP, and positively with highdensity lipoprotein cholesterol (HDLC)/total cholesterol (TC) ratio. HMW mimicked TA associations. The intervention program led to a reduction of TC, low-density lipoprotein cholesterol (LDLC), insulin, HOMA-IR, and trunk percentage of fat, and an increase of HDLC/TC ratio, in the obese group. BMI improvements prevented adiponectin reduction and correlated with increments in HMW and MMW.

CONCLUSION: Obesity-related increase in MS features might be linked to lower adiponectin. HMW and MMW were the multimers that most explained the MS features. The intervention program improved the lipid profile and IR, and prevented the reduction of adiponectin.

A diponectin is mainly secreted by adipocytes in humans $(1,2)$, but total adiponectin (TA) levels are paradoxically lower in obese children, and even lower in obese individuals with metabolic syndrome (MS) $(3,4)$.

Adiponectin improves lipid metabolism by lowering the synthesis of free fatty acid and stimulating $\beta$-oxidation (5).
Furthermore, it is inversely related with triglyceride (TG) levels and positively related with high-density lipoprotein cholesterol (HDLc) concentration. A study from our group showed that adiponectin modulates the effect of apo E genotype on lipid profile (6). Adiponectin also presents an anti-inflammatory action, e.g., it inhibits proinflammatory tumor necrosis factor- $\alpha$ (TNF- $\alpha$ ) secretion by macrophages. This activity might have an important role in insulin resistance (IR), as TNF- $\alpha$ inhibits insulin signaling $(7,8)$, while adiponectin increases insulin sensitivity (9).

In pediatric ages, adiponectin levels correlate negatively with age (6). The difference between genders is well established, with women presenting with higher adiponectin concentrations $(6,10)$. Sexual hormones might underlie sex-related changes as before puberty; no differences are found between genders (11).

The body fat distribution influences adiponectin levels, with abdominal obesity associated with lower concentrations $(4,12)$. The association between central adiposity and reduced adiponectin was seen with visceral fat accumulation in children (13) and adults (14).

Adiponectin circulates as three complexes: trimers, hexamers (two trimers), and larger structures of trimers -12 to 18 mers being referred as low, medium, and high-molecular-weight adiponectin, respectively (LMW, MMW, and HMW) (15).

The different multimers are suggested to present different biological functions, but results are still inconclusive. HMW was described as a better marker of metabolic abnormalities in obese children than TA or the other multimers (16), and to be particularly related to improved insulin sensitivity (17) and lipid profile (18).

The decrease of TA in obese children appears to be mainly due to the reduction of the HMW multimer (16). Controversially, another study found no difference between obese and lean prepubertal boys regarding total and HMW adiponectin (19).

Diet-induced weight loss in obese adults is associated with an improvement in adiponectin levels, with no further impact

'Biological Science Department, Faculty of Pharmacy, University of Oporto, Oporto, Portugal; ${ }^{2}$ Institute for Molecular and Cell Biology, University of Oporto, Oporto, Portugal; ${ }^{3}$ Research Centre in Physical Activity, Health and Leisure - CIAFEL, Faculty of Sports, University of Oporto, Oporto, Portugal; ${ }^{4}$ High Education Institute From Maia - ISMAI, Maia, Portugal; ${ }^{5}$ Health Science Research Centre, University of Beira Interior, Covilhã, Portugal; ${ }^{6}$ Children and Adolescent Centre, CUF Hospital, Center for Health Technology and Services Research (CINTESIS), Faculty of Medicine, University of Oporto, Oporto, Portugal; ${ }^{7}$ Childhood and Adolescence Department, Paediatric Service, Oporto Hospital Centre, Oporto, Portugal; ${ }^{8}$ Abel Salazar Biomedical Sciences Institute, University of Oporto, Oporto, Portugal. Correspondence: Luís Belo (luisbelo@ff.up.pt) 
from exercise (20). Contrarily, another study found no impact on TA levels after a nutritional counseling program in prepubertal Portuguese children, despite the improvement in BMI $\mathrm{z}$-score (BMIzsc) and blood lipids (21). Different results were found in a study involving obese adolescent boys, as improvements in adiponectin levels were obtained both by energy restriction and by exercise separately, moreover, cumulative effects were present when both approaches were combined (22).

The aims of this study were: (i) to clarify changes in circulating TA and related multimers in obese children and adolescents, (ii) to evaluate adiponectin relation with markers of MS, and (iii) to study the impact of regular physical exercise (PE) in adiponectin and in MS features.

\section{RESULTS}

\section{Cross-Sectional Study}

The control and obese groups were matched for sex, age, and tanner stage. The obese individuals presented a lipid profile with significant cardiovascular risk changes, namely, higher TG, low-density lipoprotein cholesterol (LDLc) and very-low-density lipoprotein cholesterol (VLDLc), and lower HDLc and HDLc/total cholesterol (TC) ratio; they also presented increased IR (measured by homeostasis model assessment of IR (HOMA-IR)) and insulin, although showing normal levels of glucose. A proinflammatory status was present in obese individuals, with lower values of adiponectin and C-reactive protein (CRP) median being almost five times higher than that of the control group (Supplementary Table S1 online).

\section{Longitudinal Study}

The variation of anthropometric variables during the study period for obese and controls is presented in Table 1. The groups did not differ regarding sex and tanner stage (data not shown). At the end of the intervention program, both groups presented a significant decrease in BMIzsc, but only the obese group presented a significant reduction of the trunk percentage of fat.

The weight loss in the obese group, as shown by the reduction in BMIzsc, was accompanied by changes in the studied biochemical variables. Obese individuals improved the lipid

Table 1. Variation of anthropometric, nutritional, and biochemical characterization during the intervention program in control and obese groups (longitudinal study, $n=57$ )

\begin{tabular}{|c|c|c|c|c|c|c|}
\hline & \multicolumn{2}{|c|}{ Control (22) } & \multirow[b]{2}{*}{$P$} & \multicolumn{2}{|c|}{ Obese (35) } & \multirow[b]{2}{*}{$P$} \\
\hline & First evaluation & Second evaluation & & First evaluation & Second evaluation & \\
\hline Height (cm) & $140.9 \pm 17.5$ & $142.4 \pm 16.8$ & $<0.001$ & $145.7 \pm 15.1$ & $148.0 \pm 14.5$ & $<0.001$ \\
\hline $\mathrm{BMI}\left(\mathrm{kg} / \mathrm{m}^{2}\right)$ & $18.4 \pm 3.4$ & $18.3 \pm 3.8$ & 0.350 & $27.1 \pm 5.4$ & $26.7 \pm 5.6$ & 0.206 \\
\hline BMI z-score & $0.21 \pm 0.79$ & $-0.04 \pm 0.96$ & 0.002 & $2.1 \pm 0.3$ & $1.9 \pm 0.5$ & 0.001 \\
\hline Waist/height & $0.472 \pm 0.048$ & $0.467 \pm 0.054$ & 0.494 & $0.607 \pm 0.065$ & $0.608 \pm 0.063$ & 0.886 \\
\hline Total $\%$ fat $^{\mathrm{a}}$ & $28.5 \pm 4.6$ & $28.9 \pm 5.6$ & 0.460 & $40.9 \pm 6.7$ & $40.7 \pm 6.0$ & 0.663 \\
\hline Trunk $\%$ fat $^{\mathrm{a}}$ & $24.1 \pm 4.8$ & $23.5 \pm 6.2$ & 0.429 & $39.8 \pm 8.2$ & $38.1 \pm 7.2$ & 0.013 \\
\hline \multicolumn{7}{|l|}{ Lipid profile } \\
\hline $\mathrm{TG}(\mathrm{mg} / \mathrm{dl})$ & $58.0(46.0-72.8)$ & $68.5(57.5-80.2)$ & 0.147 & $71.0(52.0-108.0)$ & $66.0(53.0-102.0)$ & 0.257 \\
\hline $\operatorname{VLDLc}(\mathrm{mg} / \mathrm{dl})$ & $11.6(9.2-14.6)$ & $13.7(11.6-16.0)$ & 0.145 & $14.2(10.4-21.5)$ & $13.2(10.6-20.4)$ & 0.255 \\
\hline $\mathrm{HDLC} / \mathrm{TC}$ & $0.329(0.307-0.374)$ & $0.326(0.294-0.392)$ & 0.934 & $0.293(0.231-0.327)$ & $0.319(0.251-0.348)$ & 0.004 \\
\hline \multicolumn{7}{|l|}{ Glucose metabolism } \\
\hline Glucose (mg/dl) & $84.5(77.0-90.2)$ & $74.5(70.0-83.2)$ & 0.002 & $81.0(76.0-85.0)$ & $77.0(72.0-81.0)$ & 0.001 \\
\hline Insulin $(\mu \mathrm{U} / \mathrm{ml})$ & $6.1(4.5-9.8)$ & $6.5(3.8-9.8)$ & 0.636 & $12.0(7.0-17.3)$ & $10.7(8.1-15.1)$ & 0.048 \\
\hline HOMA & $1.18(0.94-1.94)$ & $1.15(0.74-1.74)$ & 0.796 & $2.46(1.38-3.85)$ & $1.88(1.40-3.09)$ & 0.014 \\
\hline \multicolumn{7}{|l|}{ Inflammatory markers } \\
\hline $\mathrm{CRP}(\mathrm{mg} / \mathrm{l})$ & $0.32(0.24-0.88)$ & $0.60(0.21-2.19)$ & 0.083 & $2.00(1.12-3.41)$ & $1.42(0.96-3.44)$ & 0.688 \\
\hline
\end{tabular}

Results are presented mean \pm SD or median (interquartile range).

aControl, $n=19$; obese, $n=31$.

CRP, C-reactive protein; HDLc, high-density lipoprotein cholesterol; LDLc, low-density lipoprotein cholesterol; HOMA, homeostasis model assessment; TG, triglyceride; TC, total cholesterol; VLDLc, very-low-density lipoprotein cholesterol. 
Table 2. Total adiponectin and adiponectin isoforms in the control and obese groups variation with the intervention program (longitudinal study, $n=23$ )

\begin{tabular}{|c|c|c|c|c|c|c|c|c|c|}
\hline & \multirow{2}{*}{\multicolumn{3}{|c|}{ Control (10) }} & \multicolumn{6}{|c|}{ Obese } \\
\hline & & & & \multicolumn{3}{|c|}{ BMI z-score reduction $<0.2(8)$} & \multicolumn{3}{|c|}{ BMI z-score reduction $\geq 0.2(5)$} \\
\hline & $\begin{array}{c}\text { First } \\
\text { evaluation }\end{array}$ & $\begin{array}{l}\text { Second } \\
\text { evaluation }\end{array}$ & $P$ & $\begin{array}{c}\text { First } \\
\text { evaluation }\end{array}$ & $\begin{array}{l}\text { Second } \\
\text { evaluation }\end{array}$ & $P$ & $\begin{array}{c}\text { First } \\
\text { evaluation }\end{array}$ & $\begin{array}{l}\text { Second } \\
\text { evaluation }\end{array}$ & $P$ \\
\hline Adiponectin $(\mu \mathrm{g} / \mathrm{ml})$ & $4.36 \pm 1.79$ & $3.87 \pm 1.25$ & 0.185 & $4.18 \pm 1.41$ & $3.31 \pm 1.34$ & 0.008 & $4.05 \pm 1.33$ & $3.58 \pm 0.94$ & 0.133 \\
\hline $\mathrm{HMW}(\mu \mathrm{g} / \mathrm{ml})$ & $2.37 \pm 1.34$ & $2.16 \pm 0.98$ & 0.442 & $2.15 \pm 0.86$ & $1.74 \pm 0.95$ & 0.015 & $2.00 \pm 0.99$ & $1.88 \pm 0.58$ & 0.648 \\
\hline$\% \mathrm{HMW}$ & $52.1 \pm 7.7$ & $53.5 \pm 9.8$ & 0.476 & $50.6 \pm 3.9$ & $50.3 \pm 7.7$ & 0.897 & $48.1 \pm 8.1$ & $51.8 \pm 4.0$ & 0.372 \\
\hline$\% \mathrm{MMW}$ & $24.4 \pm 5.5$ & $25.0 \pm 3.4$ & 0.700 & $26.8 \pm 3.2$ & $29.2 \pm 4.6$ & 0.155 & $26.2 \pm 6.0$ & $27.8 \pm 3.1$ & 0.708 \\
\hline$\%$ LMW & $23.5 \pm 7.8$ & $21.5 \pm 7.9$ & 0.452 & $22.6 \pm 4.7$ & $20.5 \pm 7.1$ & 0.455 & $25.6 \pm 7.4$ & $20.4 \pm 4.6$ & 0.233 \\
\hline
\end{tabular}

Results are presented as mean \pm SD

HMW, high-molecular weight; LMW, low-molecular weight; MMW, medium-molecular weight.

profile, with a significant reduction of TC and LDLc concentrations, and an increase in the HDLc/TC ratio; IR was also improved, with a decrease in insulin levels and in HOMA-IR.

On their turn, controls did not present any significant changes in lipid profile or IR markers, despite the variation of BMIzsc. Levels of adiponectin were reduced in both groups during the study period.

Obese and control groups were also compared for the absolute and relative variations of the anthropometric and biochemical variables (data not shown). No difference between the two groups was found when comparing changes in anthropometric data. Conversely, an increase in $\triangle \mathrm{HDLc} / \mathrm{TC} \%$ and a trend for the lowering of HOMA-IR was seen in obese group (Supplementary Figures S1 and S2 online).

\section{Analysis of Adiponectin Multimers}

To better understand the function of adiponectin isoforms and to verify any difference between obese and controls, we selected a prepubertal population, in order to minimize the variations caused by other factors rather than obesity (e.g., sexual hormones). The control $(n=10)$ and obese $(n=13)$ groups were adjusted for gender ( $50 \%$ females vs. $46.2 \%$ females, respectively) and age (mean $7.3 \pm 1.4$ vs. $7.9 \pm 1.4$, respectively).

The differences in anthropometric and biochemical variables observed between obese and control groups in the larger population remained constant although TG, HDLc, LDLc, and VLDLc presented only a borderline statistical significance (data not shown), and adiponectin lost significance.

No differences were observed between obese and control groups regarding the absolute and relative multimer concentrations, before and after the PE program (data not shown).

We further analyzed the impact of the PE program in TA and in its multimers by dividing the obese group based on having achieved, or not, a BMIzsc reduction of 0.2 , a cutoff characterizing a moderate-to-small reduction in adiposity (Table 2).

The control group did not present significant changes in adiponectin during the intervention period. Regarding the
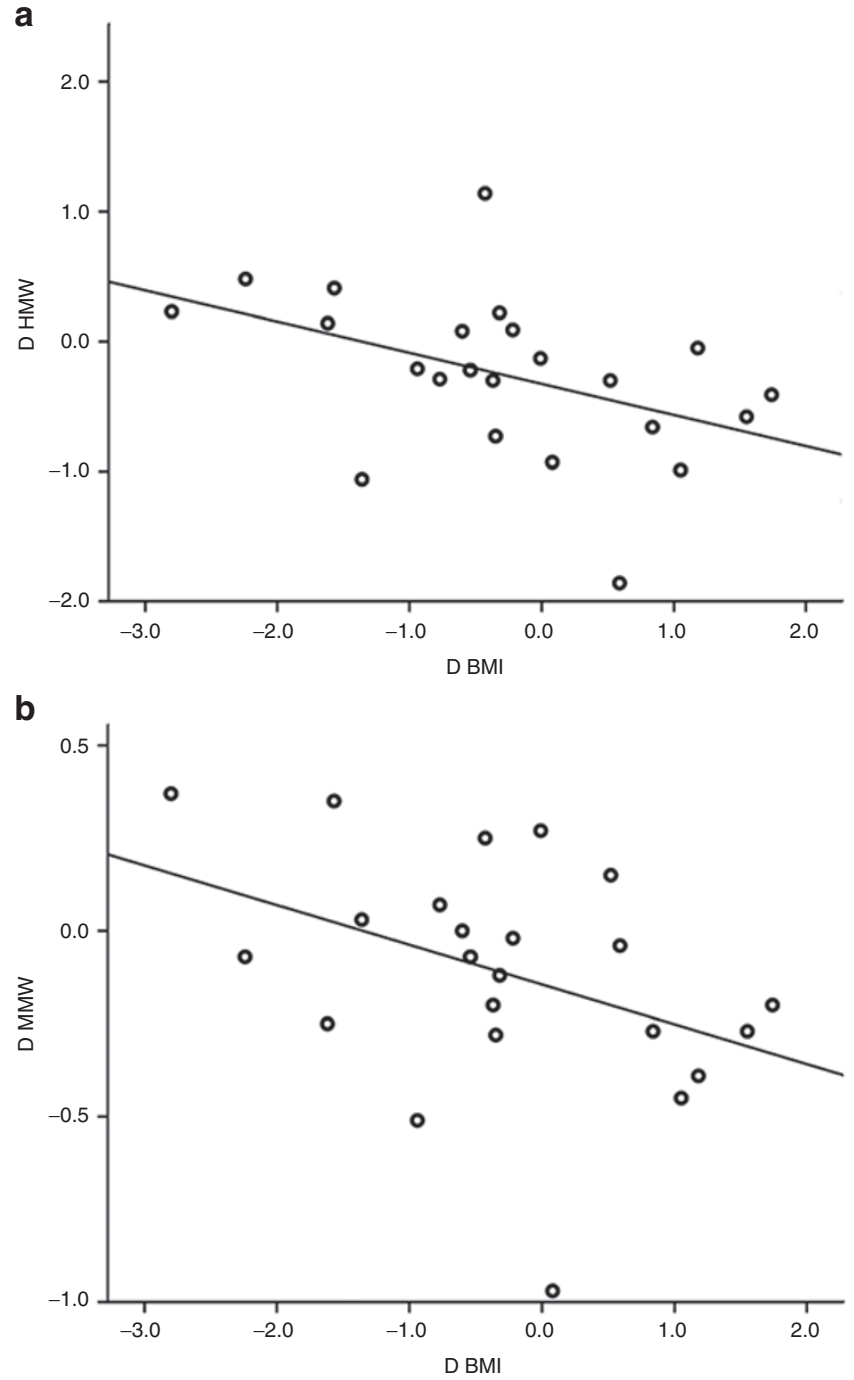

Figure 1. Variation of high- and medium-molecular-weight adiponectin (HMW and MMW) with changes in BMI after the intervention program. $D$, $\Delta$. (a) Spearman's $r=-0.543, P=0.007$; (b) Spearman's $r=-0.431, P=0.040$. 
obese participants, the group that reached the cutoff value also did not present any significant reduction of adiponectin. Conversely, obese individuals who did not reach the cutoff value presented a general reduction of TA and its multimers absolute concentration. The relative percentage of the different isoforms was maintained in all groups.

To clarify the functions of the different adiponectin multimers, several correlations were performed. First, we analyzed associations between TA and the other studied variables using the entire studied population (cross-sectional study; $n=104$ ), and then we performed subanalysis between changes in studied variables over the interventional study ( $n=57$ for TA; $n=$ 23 for adiponectin multimers). The levels of TA $(n=104)$ correlated inversely with BMI, total percentage of body fat, TG, insulin, HOMA-IR, and CRP, and positively with HDLc and HDLc/TC ratio (Supplementary Table S2 online). Regarding the longitudinal analysis $(n=57)$, changes in TA were inversely correlated with changes in markers of adiposity and in TG levels. The multimer that most mimics TA associations at the baseline, was the HMW isoform; even though, statistical significance was lost, probably due to the lower number of cases $(n=23)$.

Improvements in adiposity variables at prepubertal stages, especially BMI, correlated with increments in circulating levels of HMW and MMW forms (Figure 1). Moreover, by multiple regression analysis, the parameter that best explained changes in HMW isoform was the $\Delta$ BMIzsc $\left(R^{2}=0.299\right.$, standardized coefficient/ $\beta$ : $-0.547, P=0.013)$.

The increase in HMW relative percentage was related with lower levels of circulating TG. In contrast, despite the negative correlation with adiposity, an increased relative percentage of MMW was associated with increased levels of TC and LDLc $(P<0.01$ and $P<0.05$, respectively).

\section{DISCUSSION}

\section{Cross-Sectional Study}

Childhood obesity was accompanied by worsening of MS markers $(3,4)$. In our study, the obese group presented a worse lipid profile, increased IR (measured by HOMA-IR), and inflammation, as seen in Supplementary Table S1 online.

The obese group presented increased IR, although showing normal levels of glucose, pointing at an unbalanced glucose metabolism. In children, the insulin levels seem to be an early indicator of metabolic changes, appearing first than anomalies in glycemia.

\section{Longitudinal Study}

Although both obese and controls reduced BMI score following the intervention program, only the obese group presented clear changes in the body composition, with a significant reduction of the trunk percentage of fat. The body fat distribution influences the metabolism; actually, a central accumulation of adiposity has been associated with an increase in cardiovascular disease risk $(4,12,13)$. The reduction of the central adiposity in the obese group is an important indicator of the positive impact of the PE program.
Accompanying the decrease in adiposity in obese individuals, were the improvements in biochemical variables, namely lipid profile and insulin. Obese individuals were presented with an improved lipid profile as seen by a decrease in $\Delta \mathrm{HDLc} /$ TC\% ratio. Actually, more than $75 \%$ of the obese individuals presented a decrease in that athrogenic index (D HDLc/TC\%), while only $50 \%$ of the controls obtained the same result. This is an important finding as this ratio is an accepted marker of atherosclerotic risk (6). As well, the trend to an improvement in HOMA-IR in obese patients indicated a better glucose homeostasis; a particularly relevant point as IR continues to explain most, if not all, of MS (23). Together, these changes demonstrate a global improvement in lipid and glucose metabolisms following the intervention program.

The lack of changes seen for controls, regarding lipid profile or IR, is probably due to the fact that these variables were already at good levels and, although the practice of exercise improved the nutritional status, their metabolism was previously balanced and no further changes could be detected.

Considering that the metabolism of obese individuals is unbalanced, the BMIzsc reduction observed in this group, with a particular decrease in central adiposity, likely contributed to lead the biochemical variables to the desirable levels. In fact, changes in body fat distribution might be the key factor behind the metabolic improvement.

The PE program was unable to increase TA values in any of the groups, contrarily to other studies (22). TA and adiponectin multimers seem to require marked reductions in adiposity to improve. In fact, changes in TA, HMW, and MMW adiponectin were negatively associated with changes in BMI and BMIzsc in our study, however, the lack of significant improvements might be related with the lower reduction of BMIzsc presented by our participants, despite the significant changes in other anthropometric variables. Additionally, adiponectin is negatively correlated with age in children and adolescents, meaning that it might be necessary greater BMIzsc reductions to oppose this physiological trend (6).

\section{Analysis of Adiponectin Multimers}

For the study of adiponectin isoforms, we selected a prepubertal population in order to minimize the variations caused by other factors rather than obesity. Despite the differences in anthropometric and biochemical variables between obese and controls in the larger population were, somehow, maintained, no significant changes were observed between the two groups regarding TA and absolute and relative multimers concentrations, both before and after the PE program (data not shown). Oppositely, some studies have pointed to an increase in HMW following intervention $(16,24)$. The lack of significant differences might be related to a small sample size, however, it can also indicate that the differences previously found could be due to confounding factors, present later in life, or, that differences in TA concentrations seen at older ages, might be caused by a cumulative effect, still not evident at early ages. So, acting early might be the best strategy to avoid obesity impact later in life. 
TA and its multimers absolute concentrations did not vary both in controls and in the obese group that reached the cutoff value for BMIzsc reduction (0.2), however, in the obese individuals who did not reach that cutoff, there was noted a general reduction. Interestingly, the relative percentage of the different isoforms was maintained in all groups.

Other studies have reported that BMIzsc reduction is related to an increase in TA, particularly in the HMW form $(16,24)$. In children, adiponectin decreases with aging (6); therefore, a small reduction of BMIzsc (0.2), might not be enough to raise the TA levels, but it seems to be enough to slow the process as age-related adiponectin reduction seems to be accelerated in obese individuals. Moreover, previous data from our group demonstrated that small reductions in BMIzsc are associated with significant improvements in lipid profile and IR, markers of MS inversely related to adiponectin levels (25).

Increased TA, at baseline, was correlated with lower adiposity, inflammation and IR, and with a better lipid profile (Supplementary Table S2 online). Additionally, the longitudinal analysis showed that increases in TA are related to decreases in adiposity and TG. These results are in agreement with previous studies, showing that higher values of TA are associated with reduced body weight and improved lipid profile, inflammatory status, and insulin sensitivity (9).

An important finding of our longitudinal study was that improvements in the adiposity variables, at prepubertal stages, were correlated with the increments in circulating HMW. In fact, when multiple regression analysis was used, variations of HMW were explained by changes in BMIzsc (negative association). HMW is proposed to be the most biologically active form of adiponectin, with lower adiposity associated with higher circulating HMW (16).

HMW adiponectin has been reported to correlate closer with IR than with adiposity, however, the association between TA and HMW with IR in prepubertal children is not so clear and is said to appear only between the ages of 2 and 6 y (26). Despite the age of our prepubertal group was outside those limits (2-6 y), we did not find any association between HMW and IR. Nevertheless, the increased levels of HMW relative percentage were associated with lower TG levels. HMW relation with a better lipid profile is probably explained by the influence of this multimer in liver insulin sensitivity (18).

Thus, it seems that HMW mimic the associations of TA with adiposity and lipid profile, and is possible that this adiponectin fraction explains most of those associations.

Another multimer, MMW, presented ambiguous results. Although the increase of MMW adiponectin was associated with the improvement of BMI (Figure 1), it was also linked with a worse lipid profile. More studies are necessary to verify these relations.

The impact of the other multimers rather than HMW in obesity has been poorly explored (16); actually we are, to the best of our knowledge, the first group to describe associations between the worsening of lipid profile and increasing MMW.

The study has the following limitations: (i) the sample size is not very large and some differences might have been underestimated, and (ii) adiponectin multimers were analyzed only in prepubertal individuals, an age period when the relation between adiponectin and MS is still not clear.

Despite these limitations, the study of adiponectin multimers in pediatric patients before and after an interventional study is rare, and thus, we believe that our work may offer some important information. Moreover, this is, as far as we know, the first study on this subject in Portugal.

\section{Conclusion}

Childhood obesity is accompanied by an increase in MS features (worse lipid profile and increased IR) and a proinflammatory state. Lower adiponectin levels in obese patients are likely to be related with most of these deleterious changes; however, differences are not evident at prepubertal stages. The adiponectin multimers that mostly explained MS features at prepubertal stages were HMW and MMW.

Insulin is an early indicator of IR and should be used as an early indicator of metabolic derangement in pediatric obesity.

The PE program helped to improve the lipid profile and to decrease IR in obese individuals, though with no significant variation of TA or adiponectin multimers. Nevertheless, prepubertal patients who reduced the BMIzsc maintained adiponectin levels, contrarily to individuals who did not.

Understanding how TA and its oligomers influence the metabolism is an important issue, particularly for children, in whom an early intervention can present relevant impact in the future. Thus, our work highlights that the different circulating forms of adiponectin might present different, or even opposite effects, in MS features. On its turn, weight loss helped to sustain what appears to be a positive profile of adiponectin isoforms.

\section{METHODS}

\section{Subjects}

The study consisted of a cross-sectional and a longitudinal part. The cross-sectional study involved 104 children and adolescents (59 females), aged 5-18 y, after written informed consent was obtained from their parents. The population was recruited in: (i) two pediatric obesity outpatient clinics in Oporto and (ii) a school-based PE program in five primary and two middle and high public schools from Oporto suburban setting.

The longitudinal study consisted of an 8-mo PE program. Fiftyseven individuals completed the intervention (27 females).

\section{Procedures and Assays}

Anthropometric and clinical evaluation. Height and weight were evaluated. Waist circumference was measured at the superior border of the iliac crest (27). Obesity was defined as BMIzsc greater than +1.65 for age and gender, according to 2000 Centre for Disease Control and Prevention growth charts (28). Controls were selected from the participants at schools, after clinical evaluation, considering their BMIzsc and absence of known comorbidities. Body composition was evaluated by dual-energy X-ray absorptiometry.

Analytical and clinical data of the participants in the longitudinal study were collected before (October 2011; first evaluation) and after the intervention program (June 2012; second evaluation). Development of puberty was assessed by Tanner stages.

Physical activity program. We performed a longitudinal study, included in the "ACORDA Project", applied to young people with overweight and obesity, aimed to change behaviors by providing easy access to the practice of PA and associating food counseling and clinical supervision. 
All overweight participants were asked to modify their lifestyle habits and to participate in regular PE classes. Attendance averaged $85 \%$. The planning program converged $3 \mathrm{~h}$ of physical education and $2 \mathrm{~h}$ of after-school sessions ( $1 \mathrm{~h}$ each session) resulting in a total of $5 \mathrm{~h}$ per week, during 8 mo. Two graduates in Sport Sciences, under the guidance of two researchers, supervised the sessions, ensuring that the type and variety of exercises would be performed according to previously held planning to apply equally to all schools. PE sessions included $15 \mathrm{~min}$ for warm-up with aerobic endurance and flexibility, $30 \mathrm{~min}$ of working circuit for aerobics, strength training, coordination and balance, with balls, bows, strings, and callisthenic exercises, 10 min of games to promote the enjoyment, and 5 min of stretching. All activities were done in indoor schools' sports facilities. Exercises and games were progressively intensified as individually tolerated. Training intensity and compliance between individuals were defined to induce heart rate higher than 80 of each child's maximum heart rate. To ensure this, 10 randomly selected children wore a portable heart rate monitor during sessions (Polar Team2 Pro, Polar, Finland).

The nature, benefits, and risks of the study were explained to the volunteers, and a parent's written informed consent was obtained before the study, consistent with the Helsinki Declaration. The experimental protocol was approved by the Review Committee of the Scientific Board of the Faculty of Sport, University of Porto as well as by the Foundation of Science and Technology (FCT, Portugal).

Blood samples. After an overnight fast, blood was obtained by venepuncture in ethylenediaminetetraacetic acid-containing tubes and processed within $2 \mathrm{~h}$ of collection. Aliquots of plasma were made and stored at $-80^{\circ} \mathrm{C}$ until assayed.

Biochemical analysis. TC, TG, HDLc, glucose, insulin, and CRP were measured using automated technology, as described elsewhere (25). LDLc and VLDLc were calculated using Friedewald formula (29). HOMA-IR was determined according to Matthews (30).

\section{Adiponectin}

TA. Plasma concentration of TA was evaluated by a commercial enzyme-linked immunoassay (e-Bioscence, San Diego, CA).

Adiponectin multimers. Absolute and relative plasma concentration of adiponectin circulating multimers (HMW, MMW, and LMW) was determined using a commercial available kit (Alpaco, Salem, NH).

\section{Exclusion Criteria and Ethical Approval}

Smokers, subjects with reduced mobility, under regular medication or with diabetes mellitus, endocrinology disorders, hereditary, inflammatory, or infectious diseases were excluded from the study.

The protocol was approved by the Hospitals Committees of Ethics and the Regional Education Board, and schools agreed to participate.

\section{Statistical Analysis}

Statistical analysis was performed using the Statistical Package for Social Sciences (SPSS software, version 20, IBM, Armonk, NY). Kolmogorov-Smirnov analysis was used to test if the results were normally distributed. Results normally distributed are presented as mean $\pm \mathrm{SD}$, otherwise are presented as median (interquartile range). Variables which distribution differed from normal were transformed for better fitting. Distributions were analyzed using $\chi^{2}$ test and Fisher's exact test.

Differences between groups were assessed using Student's $t$-test. The strength of the associations was estimated by Spearman correlation coefficient. Significance was accepted at $P<0.05$.

\section{SUPPLEMENTARY MATERIAL}

Supplementary material is linked to the online version of the paper at http:// www.nature.com/pr

\section{ACKNOWLEDGMENTS}

The authors thank all participants and parents for their collaboration and the nurses and laboratory technicians of the hospitals involved for their technical support. We also thank the ACORDA team for all the help in the intervention program.

\section{STATEMENT OF FINANCIAL SUPPORT}

This work was funded by the European Regional Development Fund (FEDER, Europe) through the Operational Competitiveness Programme (COMPETE, Portugal) and by National Funds through Fundação para a Ciência e a Tecnologia (FCT, Portugal) under the project FCOMP-01-0124FEDER-028613 (PTDC/DTP-DES/0393/2012). A PhD grant was attributed to H. Nascimento by Fundação para a Ciência e a Tecnologia (FCT, Portugal) (SFRH/BD/61407/2009).

Disclosure: The authors declare that there are no conflict of interest to disclose.

\section{REFERENCES}

1. Maeda K, Okubo K, Shimomura I, Funahashi T, Matsuzawa Y, Matsubara K. cDNA cloning and expression of a novel adipose specific collagenlike factor, apM1 (AdiPose Most abundant Gene transcript 1). Biochem Biophys Res Commun 1996;221:286-9.

2. Saito K, Tobe T, Minoshima S, et al. Organization of the gene for gelatinbinding protein (GBP28). Gene 1999;229:67-73.

3. Calcaterra V, De Amici M, Klersy C, et al. Adiponectin, IL-10 and metabolic syndrome in obese children and adolescents. Acta Biomed 2009;80:117-23.

4. Yoshinaga M, Takahashi H, Shinomiya M, Miyazaki A, Kuribayashi N, Ichida F. Impact of having one cardiovascular risk factor on other cardiovascular risk factor levels in adolescents. J Atheroscler Thromb 2010;17:116775.

5. Xu A, Wang Y, Keshaw H, Xu LY, Lam KS, Cooper GJ. The fat-derived hormone adiponectin alleviates alcoholic and nonalcoholic fatty liver diseases in mice. J Clin Invest 2003;112:91-100.

6. Nascimento H, Silva L, Lourenço P, et al. Lipid profile in Portuguese obese children and adolescents: interaction of apolipoprotein E polymorphism with adiponectin levels. Arch Pediatr Adolesc Med 2009;163:1030-6.

7. Maeda N, Takahashi M, Funahashi T, et al. PPARgamma ligands increase expression and plasma concentrations of adiponectin, an adipose-derived protein. Diabetes 2001;50:2094-9.

8. Bruun JM, Lihn AS, Verdich C, et al. Regulation of adiponectin by adipose tissue-derived cytokines: in vivo and in vitro investigations in humans. Am J Physiol Endocrinol Metab 2003;285:E527-33.

9. Nadeau KJ, Zeitler PS, Bauer TA, et al. Insulin resistance in adolescents with type 2 diabetes is associated with impaired exercise capacity. J Clin Endocrinol Metab 2009;94:3687-95.

10. Urbina EM, Khoury P, Martin LJ, D’Alessio D, Dolan LM. Gender differences in the relationships among obesity, adiponectin and brachial artery distensibility in adolescents and young adults. Int J Obes (Lond) 2009;33:1118-25.

11. Reinehr T, Roth C, Menke T, Andler W. Adiponectin before and after weight loss in obese children. J Clin Endocrinol Metab 2004;89: 3790-4.

12. Cnop M, Havel PJ, Utzschneider KM, et al. Relationship of adiponectin to body fat distribution, insulin sensitivity and plasma lipoproteins: evidence for independent roles of age and sex. Diabetologia 2003;46:459-69.

13. Ogawa Y, Kikuchi T, Nagasaki K, Hiura M, Tanaka Y, Uchiyama M. Usefulness of serum adiponectin level as a diagnostic marker of metabolic syndrome in obese Japanese children. Hypertens Res 2005;28:51-7.

14. Kwon K, Jung SH, Choi C, Park SH. Reciprocal association between visceral obesity and adiponectin: in healthy premenopausal women. Int J Cardiol 2005;101:385-90.

15. Schober F, Neumeier M, Weigert J, et al. Low molecular weight adiponectin negatively correlates with the waist circumference and monocytic IL-6 release. Biochem Biophys Res Commun 2007;361:968-73.

16. Araki S, Dobashi K, Kubo K, Asayama K, Shirahata A. High molecular weight, rather than total, adiponectin levels better reflect metabolic abnormalities associated with childhood obesity. J Clin Endocrinol Metab 2006;91:5113-6.

17. Liu Y, Retnakaran R, Hanley A, Tungtrongchitr R, Shaw C, Sweeney G. Total and high molecular weight but not trimeric or hexameric forms of adiponectin correlate with markers of the metabolic syndrome and liver injury in Thai subjects. J Clin Endocrinol Metab 2007;92:4313-8. 
18. Neumeier M, Sigruener A, Eggenhofer E, et al. High molecular weight adiponectin reduces apolipoprotein $\mathrm{B}$ and $\mathrm{E}$ release in human hepatocytes. Biochem Biophys Res Commun 2007;352:543-8.

19. Murdolo G, Nowotny B, Celi F, et al. Inflammatory adipokines, high molecular weight adiponectin, and insulin resistance: a population-based survey in prepubertal schoolchildren. PLoS One 2011;6:e17264.

20. Christiansen T, Paulsen SK, Bruun JM, Pedersen SB, Richelsen B. Exercise training versus diet-induced weight-loss on metabolic risk factors and inflammatory markers in obese subjects: a 12-week randomized intervention study. Am J Physiol Endocrinol Metab 2010;298:E824-31.

21. Pedrosa C, Oliveira BM, Albuquerque I, Simões-Pereira C, Vaz-deAlmeida MD, Correia F. Metabolic syndrome, adipokines and ghrelin in overweight and obese schoolchildren: results of a 1-year lifestyle intervention programme. Eur J Pediatr 2011;170:483-92.

22. Elloumi M, Ben Ounis O, Makni E, Van Praagh E, Tabka Z, Lac G. Effect of individualized weight-loss programmes on adiponectin, leptin and resistin levels in obese adolescent boys. Acta Paediatr 2009;98:1487-93.

23. Eckel RH, Alberti KG, Grundy SM, Zimmet PZ. The metabolic syndrome. Lancet 2010;375:181-3.

24. Araki S, Dobashi K, Yamamoto Y, Asayama K, Kusuhara K. Increased plasma isoprostane is associated with visceral fat, high molecular weight adiponectin, and metabolic complications in obese children. Eur J Pediatr 2010;169:965-70.
25. Nascimento H, Costa E, Rocha-Pereira P, et al. Cardiovascular risk factors in portuguese obese children and adolescents: impact of small reductions in body mass index imposed by lifestyle modifications. Open Biochem J 2012;6:43-50.

26. Ibáñez L, Lopez-Bermejo A, Diaz M, Angulo M, Sebastiani G, de Zegher F. High-molecular-weight adiponectin in children born small- or appropriate-for-gestational-age. J Pediatr 2009;155:740-2.

27. Center for Disease Control and Prevention. National Health and Nutrition Examination Survey (NHANES) - Anthropometry Procedures Manual, 2009. http://www.cdc.gov/nchs/data/nhanes/nhanes_09_10/BodyMeasures_09.pdf.

28. Cole TJ, Bellizzi MC, Flegal KM, Dietz WH. Establishing a standard definition for child overweight and obesity worldwide: international survey. BMJ 2000;320:1240-3.

29. Friedewald WT, Levy RI, Fredrickson DS. Estimation of the concentration of low-density lipoprotein cholesterol in plasma, without use of the preparative ultracentrifuge. Clin Chem 1972;18:499-502.

30. Matthews DR, Hosker JP, Rudenski AS, Naylor BA, Treacher DF, Turner RC. Homeostasis model assessment: insulin resistance and betacell function from fasting plasma glucose and insulin concentrations in man. Diabetologia 1985;28:412-9. 